WSSR \#894356, VOL 0, ISS 0

\title{
Preferences and Expectations for Delivering Bad News Among Korean Older Adults
}

\author{
Eunjeong Ko, Holly Nelson-Becker, Minjung Shin, and Young Joon Park
}

\section{QUERY SHEET}

This page lists questions we have about your paper. The numbers displayed at left can be found in the text of the paper for reference. In addition, please review your paper as a whole for correctness.

Q1. Au: Confirm all authors, affiliations, and correspondence information appear correctly.

Q2. Au: Please specifically mention Table 1 in text.

Q3. Au: Is shortened title OK? It must be 50 characters or fewer.

Q4. Au: Lee et al., 2012, is listed in the References as having been published in 2013. Please confirm the correct year of publication and revise or add where necessary.

Q5. Au: Please provide the information where you have written "BLINDED FOR REVIEW."

Q6. Au: Please confirm that $\$ 7,500$ is in US dollars.

Q7. Au: Please confirm the publisher for Beauchamp \& Childress, 1994 - should it be Oxford University Press?

Q8. Au: Please cite Berkman \& Ko, 2009, in text or remove from the References.

Q9. Au: Please cite Fujimori et al., 2007, in text or remove from the References.

Q10. Au: Please confirm the page range for Kirk et al., 2004.

\section{TABLE OF CONTENTS LISTING}

The table of contents for the journal will list your paper exactly as it appears below:

Preferences and Expectations for Delivering Bad News Among Korean Older Adults

Eunjeong Ko, Holly Nelson-Becker, Minjung Shin, and Young Joon Park 


\section{Preferences and Expectations for Delivering Bad News Among Korean Older Adults}

\section{INTRODUCTION}

Disclosing a serious illness such as cancer is a complex process that involves a patient's emotional, psychological, social, and cultural con5 structions (Blackhall, Frank, Murphy, \& Michel, 2001; Fujimori et al., 2005; Gold, 2004). Com- municating unfavorable news to a patient is a difficult task as it often produces devastating emotional effects on patients and family members (Elwyn, Fetters, Sasaki, \& Tsuda, 2002; Fielding, Wong, \& Ko, 1998). Hence, informing patients about their terminal status needs to involve more than whether or not to share the

Eunjeong Ko, Ph.D., is an Assistant Professor at San Diego State University, School of Social Work, San Diego, CA.

Holly Nelson-Becker, Ph.D., is a Professor at Loyola University, School of Social Work, Chicago, IL.

Minjung Shin, MA, is an Assistant Professor at Daegu Polytechnic College University, Department of Social Welfare, Daegu Metropolitan, South Korea.

Young Joon Park, Ph.D., is an Assistant Professor at Daegu University, Department of Social Welfare, Gyeongbuk, South Korea.

Address correspondence to: Eunjeong Ko, Ph.D., San Diego State University, School of Social Work, 5500 Campanile Drive, San Diego, CA 92182 (E-mail: eko@mail.sdsu.edu). 
TABLE 1. Participant Characteristics

\begin{tabular}{|c|c|c|}
\hline Characteristics & $N=70$ & $\% /$ Mean $(S D)$ \\
\hline Age & 70 & $73(7.5)$ \\
\hline \multicolumn{3}{|l|}{ Gender } \\
\hline Female & 50 & $71.4 \%$ \\
\hline Education & 70 & $5.7(5.7)$ \\
\hline \multicolumn{3}{|l|}{ Income } \\
\hline Less than $\$ 7,500$ & 38 & $65.5 \%$ \\
\hline$\$ 7,501-\$ 15,000$ & 5 & $8.6 \%$ \\
\hline$\$ 15,001-\$ 30,000$ & 10 & $17.2 \%$ \\
\hline More than $\$ 30,000$ & 5 & $8.6 \%$ \\
\hline \multicolumn{3}{|l|}{ Marital status } \\
\hline Widowed & 39 & $55.7 \%$ \\
\hline Married/living together & 26 & $37.1 \%$ \\
\hline Divorced & 5 & $7.1 \%$ \\
\hline \multicolumn{3}{|l|}{ Religion } \\
\hline Buddhist & 31 & $44.3 \%$ \\
\hline Protestant & 19 & $27.1 \%$ \\
\hline Catholic & 9 & $12.9 \%$ \\
\hline Other & 11 & $15.7 \%$ \\
\hline \multicolumn{3}{|l|}{ Religiosity/Spirituality } \\
\hline Deeply religious/spiritual & 3 & $4.5 \%$ \\
\hline Fairly religious/spiritual & 15 & $22.4 \%$ \\
\hline Only slightly religious/spiritual & 35 & $52.2 \%$ \\
\hline Not at all religious/spiritual & 14 & $20.9 \%$ \\
\hline \multicolumn{3}{|l|}{ Health status } \\
\hline Very good/good & 30 & $40 \%$ \\
\hline Fair & 20 & $28.6 \%$ \\
\hline Poor & 22 & $31.4 \%$ \\
\hline \multicolumn{3}{|l|}{ Current medical diagnosis } \\
\hline Arthritis & 37 & $52.9 \%$ \\
\hline Osteoporosis & 28 & $40 \%$ \\
\hline Diabetes & 18 & $25.7 \%$ \\
\hline Hypertension & 16 & $22.9 \%$ \\
\hline Cancer & 2 & $2.9 \%$ \\
\hline
\end{tabular}

condition of their illness. Instead, questions reterminal status needs to be shared and when, as well as how and to whom the information should be delivered become salient (Clayton, Butow, \& Tattersall, 2005; Kirk, Kirk, \& Kristjanson,

20 2004; Mauri, Vegni, Lozza, Parker, \& Moja, 2009; Miyata, Takahashi, Saito, Tachimori, \& Kai, 2005; Shahidi, 2010).

The concept of autonomy in bioethics has gained primacy in medical decision making and in fact is primary of the four accepted medical ethics principles, which also include nonmaleficence (do no harm), beneficence (do good), and justice (Beauchamp \& Childress, 1994). For individuals to exercise self-determination, disclos- nosis is a necessary first step. However, the priority in bioethics might be weighted on "do no harm" for patients representing some other cultures, especially Asian (Gold, 2004; Jotkowitz, Glick, \& Gezundheit, 2006). Although disclosing terminal illness is considered to be a standard practice in Western culture, it is not readily accepted in non-Western cultures (Gongal, 2006; Jotkowitz et al., 2006; Mitchell, 1998; Shahidi, 2010). By contrast, this disclosure is considered harmful for the patient (Blackhall et al., 2001; Carrese \& Rhodes, 1995). Hence, minimizing harm takes precedence over autonomy such that nondisclosure or partial disclosure of terminal illness is accepted and even preferred in many global and indigenous populations (Carrese \& Rhodes, 1995; Miyata et al., 2005; Surbone, Ritossa, \& Spagnolo, 2004). A practical approach to decision making has been proposed by a philosopher, a medical internist, and a lawyer (Jonsen, Siegler, \& Winslade, 2010). They suggest the following questions be asked in considering case practice: 1) What are the indications for medical intervention? 2) What are the preferences of the patient? 3) What quality-oflife circumstances need to be considered? And 4) what are the contextual features in the case under review? In this model, Items 2 and 4, the preferences of the patient and the contextual features in the case, both point to an appreciation of and consideration of cultural differences that can add a layer of complexity especially to situations where the treatment team and the patient may be members of different ethnic groups holding disparate end-of-life planning assumptions.

Many studies have explored the cultural aspects in communicating bad news, yet no consensus on what is optimal or particularly detrimental has been reached. Each culture has its own unique values, so beliefs, ideas, or preferences for disclosing terminal information are divergent (Kai, Beavan, \& Faull, 2011; Mitchell, 1998; Windsor et al., 2008). Disclosure of serious illness has drawn global attention, and understanding cultural difference has become timely. Understanding unique cultural positions in disclosing terminal illness and expectations in medical decision-making practice will be more important with diverse populations. In this article, we present issues 
concerning disclosing serious illness to Korean older adults.

\section{END-OF-LIFE DECISION MAKING AND CULTURE}

There has been a growing recognition that communication is a vital component in patient care and that ineffective communication, in particular when informing a patient about the terminal nature of an illness, can yield grave consequences for patients and family members (Ahrens, Yancey, \& Kollef, 2003). Lack of clear

115 understanding about the typical course of a disease may lead to confusion in end-of-life decision making and to higher costs for care. Family members in minority cultures may feel an increased burden to do everything possible to cure their loved one and thus resist use of palliative care and hospice services. Therefore, disclosing terminal illness has long been deliberated as well as contested across cultures, particularly in the context of cancer care (Oliffe, Thorne, Hislop, \& Armstrong, 2007).

Compared with Western culture where disclosure of serious illness by medical practitioners is generally seen as best practice, the direct or full disclosure of the severity of the illness

130 to patients is not commonly practiced in Korea (Oh et al., 2004). A recent study with Korean patients and family (Yun et al., 2010) revealed that only $58 \%$ of patients as compared with $83.4 \%$ of family knew of their terminal status. While $69 \%$

135 of family was informed by a physician about the patient's health condition, only $56 \%$ of patients were directly told by a physician. Further, only $10.7 \%$ of patients reported being told by their family members. Another study done in Korea

140 (Oh et al., 2004) provided a similar finding that $86 \%$ of patients knew that they had cancer but only $37 \%$ of patients had an accurate knowledge about the stage of their cancer.

Cultural traditions about decision making in

145 Korea explain the differences in perceptions of the role of autonomy between Western and Eastern cultures. Unlike the Western cultural focus on individual autonomy, shared decision making or family-centered decision making is common in Korea (Lee et al., 2012). The role of the family 150 in medical decision making is significant: Permitting family to make decisions on behalf of their loved ones even when the patients are cognitively capable is accepted practice in Korean culture. Fear of causing emotional distress to pa- 155 tients as well as impairing their hope to fight the illness (Berkman \& Ko, 2010; Blackhall et al., 1999) are concerns that come with disclosure of the severity of one's illness. In an attempt to protect patients, family shields patients from the 160 negative information. For example, Oh and colleagues (2004) found that about $15 \%(n=17)$ of the 114 participating family members in their Korean sample refused to discuss the subject of end-of-life care with patients and $83(85.6 \%)$ of 165 97 available family participants did not allow the researchers (physicians) to approach the patients to discuss their life-sustaining treatment preference.

Despite cultural positions and traditions that 170 resist disclosure of serious illness, there is a growing need for patients to make informed decisions. According to Yun and colleagues (2004), $96.1 \%$ of patients and $76.9 \%$ of family members in Korea believed that patients do 175 need to be told their status of having a terminal illness. In addition, about $72 \%$ of the patients and $44 \%$ of the family members preferred that patients be told immediately after the diagnosis is made. Previous studies emphasize that dis- 180 closure of terminal illness is more than simply telling or not telling the truth. The conditions and timing for appropriate disclosure and the approach to delivering such news are all important to consider (Berkman \& Ko, 2010; Fujimori 185 et al., 2005; Yun et al., 2004).

While global interest in disclosing serious illness is heightened for transparency in relationships and informed decision making and care, concomitant with the increasing diversity within 190 nations, the need for understanding cultural differences in the application of bioethical principles is also critical (Oliffe et al., 2007). National Association of Social Workers (NASW) standards for palliative and end-of-life care (2013) 195 suggest that universality-the duty to take actions that hold for everyone, regardless of time, place, or people involved-and veracity-the duty to tell the truth - are both key guides for 

Because they work on the front lines in health care settings, social workers facilitate meeting patients' needs in multiple aspects: psychological, social, spiritual, and cultural (Gwyther et al., . An increasingly diverse population in the United States heightens the need for culturally competent social work practice. Gaining knowledge about communication preferences, general expectations about delivery of unfavorable news, traditions, values, and family systems in nonWestern cultures may assist these social workers to practice with greater cultural sensitivity and be better prepared to individualize care.

Nonetheless, very little is known about perspectives and preferences in the disclosure of terminal illness across cultures. A few quantitative studies (Mo et al., 2012; Yun et al., 2004, 2010,2011 ) have examined preferences and attitudes toward disclosing cancer status in Korea, which broadens understanding about prevalence and needs in disclosure of terminal illness. However, there is a dearth of in-depth qualitative information regarding to what extent and how Korean participants expect unfavorable news to be delivered to patients. This study aimed to explore Korean older adults' perspectives toward disclosing terminal illness and their expectations for delivering bad news.

\section{METHODOLOGY}

\section{Study Design and Sampling Procedure}

This study used a qualitative method. Korean older adults aged 65 years and older who were members of senior centers in Korea were interviewed in person using a semistructured interview guide. Letters describing the purpose of the study and the institutional review board (IRB)-approved procedures were sent to both directors at the selected study sites in early 2009. Follow-up phone calls were made to the directors for permission to conduct the study. During programming sessions, the staff at the study sites informed potential participants about the study. The staff members were asked to create the sampling frame for the two study sites: a a senior social welfare center in an urban city of South Korea. The third and fourth authors selected the participants at the senior social welfare center. All 40 seniors who enrolled in the senior programs in the community-based social 250 work organization gave their consent and were interviewed. Another 30 participants were randomly selected from the list of senior members at a senior social welfare center.

The staff members provided the invitation let- 255 ter to the selected participants and facilitated participation by reading the invitation and providing further explanation to those who required assistance. Inclusion criteria were adults aged 65 years and older who were cognitively compe- 260 tent and who self-identified as Korean nationality. The participants' mental status was screened by the staff members at the organization who were in contact with these seniors on a daily basis. The total sample size was 70 .

Given that standard IRB protocols were not established in Korea, all research procedures and protocols were followed by the IRB at the primary author's institution [BLINDED FOR REVIEW] (IRB \#258032). Coauthors (third, 270 Q5 fourth) who collected the data in Korea obtained an IRB certificate and adhered to IRB guidelines. Written informed consent was obtained from the participants prior to the interview.

\section{Data Collection}

Data were collected using face-to-face interviews between February 2009 and May 2009. Interviews were conducted by trained social work students in a private room at each of study sites. Prior to the qualitative interview, partic- 280 ipants were asked questions regarding demographics and health status. Each qualitative interview lasted about 20 to $30 \mathrm{~min}$. To ensure confidentiality, the participants' names and any identifiable information were omitted for analy- 285 sis and instead unique ID numbers were given. Names and unique IDs were kept in a separate file. The recorded tapes were marked with the unique ID numbers. The questionnaire and the recorded tapes were kept in a secured, locked 290 cabinet in the third and fourth authors' office in Korea. 
Recorded interviews were transcribed in Korean by the research assistants, and the third and fourth authors randomly checked the transcribed material to ensure that no information was omitted. Then the first author translated the transcription to English. Back translation was not performed in this study, but particular areas that had awkward wording or unclear phrasing identified by an English-speaking coauthor were provided to another Korean bilingual professional who acted as a consultant to ensure the accuracy of translation.

\section{Measures}

Sociodemographic variables included age, gender, income, marital status, religious affiliation, and religious/spiritual propensity. Income was measured by asking participants their combined annual household income. Religious/spiritual propensity was measured by a single item assessing how religious/spiritual the participants thought they were. The response categories included $1=$ deeply religious/spiritual

315 to $4=$ not at all religious/spiritual. Participants' health status was measured by self-report on a global health rating. Participants were also asked if they had ever been diagnosed with arthritis, osteoporosis, diabetes, hypertension, or cancer.

320 An interview guide was developed based on existing literature (Blackhall et al., 1999, 2001; Elwyn et al., 2002) and the previous experience of the first author in her research. Qualitative interviews included the following key questions,

325 among others: a) When a patient has a serious illness (e.g., cancer) and there is no chance of recovery, do you think the doctor should tell the patient and family about it? b) In what way do you think they should/should not provide infor-

330 mation about the illness? And c) how do you think the doctor should deliver the bad news? Responses were analyzed using analytic techniques detailed in the following section.

\section{Data Analysis}

335 Qualitative methods are especially useful in capturing the complexity and variety of responses to a particular question (Creswell \& Clark, 2007; Denzin \& Lincoln, 2000). A grounded theory approach was used to look for common themes in the data (Strauss \& Corbin, 340 1990). In this method, all of the data were examined to look for frequently used phrases and ideas that emerged across the respondents. Thus, a coding scheme was developed inductively from the data rather than being imposed on the data. 345 This is referred to by Strauss and Corbin (1990) as open coding. Major and minor thematic areas were then identified (usually referred to as axial coding), and these were compared across participant responses. Finally, principle themes were 350 organized into categories in a selective coding process. All of the data were transcribed and then translated into English prior to analysis. The analyst read the data multiple times to assess both narrative context and word usage with care to 355 check results with the primary author. Certain phrases that came up often-for example, what to do "when there is no hope..." and "informing about serious illness"-were used as theme identifiers. When categories were formed, the 360 methodologist checked them in reverse order to ensure that each category had sufficient support.

In qualitative analysis, it is important to confirm rigor and trustworthiness. While quantitative research has reliability and validity mea- 365 sures, these are also present in a different form in qualitative research. The reader should have confidence that the results are not particular to one researcher's interpretation of participant responses. One way to achieve this is through trian- 370 gulation with multiple analysts (Patton, 2002). In this case, the primary author served to verify the data. She read the analyst's coding scheme and confirmed the results. In addition, prolonged engagement was used to increase knowledge about 375 organizational culture and build a trusting relationship with the study population (Lincoln \& Guba, 1985). The third and fourth authors met with the directors at both sites and answered questions about the study after letters introduc- 380 ing the study were sent. This process helped the researchers to increase their familiarity with the social settings where the participants engaged in their activities. Another method used to increase credibility was use of the entire census in the first 385 location and random sampling in the second site. Although the qualitative study does not aim to increase representativeness, a random sampling 
method helps to gain knowledge from the larger group of participants (Shenton, 2004). As unknown influences are distributed across the selected samples, this minimizes bias in results and allows participants with different characteristics to voice their beliefs and perspectives (Shenton, 2004). When people begin giving the same or similar types of views, then saturation has been achieved and the researchers can be fairly confident that the boundaries of thinking around the issue have been reached.

\section{e) disclosure of serious illness as a relational}

consideration of patient coping responses, and process.

\section{Inform the Patient Directly}

430 The majority of respondents $(n=43)$ believed that informing patients directly about a potentially terminal illness is important. Clearly, the need for transparency in knowledge about the expected disease course and length of time one might live was valued. Three respondents rea- 435 soned that when a patient is aware he or she will die soon, the patient will not exhaust needless energy on aggressive care measures. Not being accurately informed has the potential to lead patients to act based on incomplete or inaccurate 440 information. Two participants expressed the perspective that it was OK to "give up" (not pursue aggressive treatment) to focus on the needs at hand.

It is better to inform patients when there 445 is no chance for recovery.... Informing them abruptly ('you will die soon') is too cruel, but I am not sure how partial information would be understood by patients. They need to be informed. Leaving it up to 450 family to let patients know is not good.

\section{Decision Making About Treatment}

When patients are given information by their physician about the nature and expectations surrounding their illness, their decisions are viewed 455 as being more appropriate to their needs: "If I have cancer, I want to know. It allows me to make a wise treatment decision." Another respondent suggested, "Informing patients directly about the situation is better. Patients and their family need 460 to be aware when there is no possibility of a [good quality of life] so that they do not prolong life with further treatment." Patients who share in an accurate assessment of their physical illness or condition are more likely to make plans that 465 are helpful to them and to exercise autonomy in the decision-making process.

\section{Planning and Preparation for the Future}

Planning for how they want to spend their remaining time based on the most accurate in- 470 formation available resonated with participants. One participant recommended informing the patient and family so they could make decisions about final acts such as burial or cremation. Further, it might be important to "leave a will or 475 ask for favors." Another stated, "When there is no hope, patients must be informed so they can make decisions-knowing in advance would 
help them to complete unfinished business such

480 as taking care of finances." This participant affirmed a desire for autonomy in end-of-life planning: "Patients need time to complete unfinished things and to end their life [in the way they choose]." Further, "both patients and their fam-

485 ily need time to arrange the remaining time [to the greatest benefit for them both]." "In general, people who are sick usually know about their status. They should know so they can prepare [for end of life]." Another individual eloquently

490 expressed the need for knowledge in this way: "As our living time was long, time to say goodbye needs to be sufficient." Overall, 15 people identified the necessity for terminally ill patients to be able to prepare for and complete unfinished

495 business. They can only do this in an environment where full disclosure is supported.

\section{Need for Use of an Ethical Standard}

Nine study participants identified an ethical dimension to being truthful with patients: "A patient needs to be informed-isn't it the right thing to do?" Other participants discussed the need for ethical standards under a range of conditions: "It is desirable for individuals to know about their situations. Doctors should inform the

505 patient first and then inform family members if a patient is not conscious." "A patient himself/herself needs to have accurate knowledge about what [the situation] is." One participant did suggest that it was important to consider po-

510 tential outcomes in deciding what to say to a patient: "Telling the truth instead of concealing the news is better. Unless concealing the information results in a positive effect, it is better to inform a patient about the situation as it is."

\section{Consideration of Patient Coping Responses}

Study participants acknowledged that the impact of news of a terminal illness could be difficult, and thus, the manner in which it was shared was vital to maintain harmony. It is crucial that patients be psychologically ready. Medical staff should take a leading role in learning ways to deliver undesirable news: "Suddenly delivering this information without any notice can be shocking for a patient, which may result in negative outcomes. Thus, I think patients need 525 to be informed after first being prepared so they can have a sense of peace." Not to share crucial health information could cause "misunderstanding" and even "hatred." Another older adult surmised,

Informing patients abruptly might cause an unexpected situation. Delivering this news in a way where they will sense it naturally ... helping them to understand naturally is better... Concealing information 535 from patients can be confusing - the health care proxy and patient need to prepare. Someday they will all know, won't they?

Other participants focused on the need for patients to practice emotion-focused coping or 540 develop their own best response capability: "A patient has a right to know. He/she might be surprised at the beginning but needs to face the situation and accept it." A patient should be informed of the truth even if it would cause pain: 545 "[He] needs to think and plan." Seven participants distinguished the need for psychological readiness and preparedness, which could be facilitated by medical professionals and their own personal coping response.

\section{Disclosure of Serious Illness as a Relational Process}

Individuals at the end of their lives do not generally live apart from a social network, though they differ on the density and reach of their net- 555 works. Family members are viewed as a significant component of that network, so the importance of sharing difficult news with family is vital because of their investment in that relationship, their compassion, and concern. Twenty- 560 three respondents suggested that family should be given information about a terminal illness in addition to the patient. One recommended a specific progression: The patient should be told first, followed by the family.

\section{Inform the Patient Indirectly}

The majority of respondents clearly favored direct involvement of the patient. The news of 
their terminal illness should be shared directly, engh some attention and care to delivery in a sensitive manner should be employed so that the patient is not caught without defenses or without support. However, three respondents voiced their preference for sharing this type of information in an indirect manner: "Informing a patient, but only indirectly, is needed. [This is to] minimize shock and also to help him/her to actively cope with it." "It is desirable to let them know about [their diagnoses]. However, if one is disappointed, his/her illness might get worse, so it is better to inform them indirectly."

\section{Inform Only the Family}

Of the respondents in this study, 11 would choose to tell only the family members and not the patient about the patient's terminal illness. Most of the reasons given for this choice were to protect the patient from pain: "If there is no hope, it is better not to inform a patient. He/she needs to be treated, but not told about his/her illness. It would aggravate pain for the one who is dying." Although respondents who advocated telling the patient saw it as a chance to resolve unresolved issues and to prepare for dying, these respondents took a more fatalistic viewpoint: "Even if a patient knows, there is nothing he or she can do." Still others felt that it would be difficult for the relationship if both the family members and patient knew the terminal prognosis. How would they speak about it together? "It would be painful for family to act as if they and I don't know anything about the illness. Thus, it is better for family to know, not patients." Two other respondents expressed similar ideas: "I want to die without knowing it due to my age. My family needs to "Wow, but I don't want to be informed"; and "What good is it for patients to know before they die? Dying without knowing anything is better." One study participant explained at greater length his concerns with breaking bad news:
It is better not to tell a patient. A patient would find out naturally when he goes to the hospital for treatment. Usually no one gives early notice to a patient. It is better for the patient to know later than know it from the beginning [of the illness]. The patient would be hurt and have a hard time when being told at first. Family needs to know, but not patients. It would make the situation worse. People die knowing or not knowing [their prognosis]. I think it is better to die 620 without knowledge of it.

Another respondent advocated that the physician should first disclose the information to the patient's family who could then decide whether to tell the patient or not. Presumably, this is 625 because family members, knowing the patient well, might understand in advance how a patient would react on hearing this news. Finally, one respondent encouraged the news to go "only to the family, not to the patients. [This will] help pa- 630 tients to have a comfortable time remaining and to say goodbye. This is believed to be good."

\section{DISCUSSION}

Increasingly, it is important to learn about cultural nuances of advance care communication 635 and planning for end-of-life treatment. Ignoring this can easily lead to miscommunication, misunderstanding, and overspending on ineffective aggressive treatment near the end of life when indications are strong that the illness will lead 640 to death. Although social work practice accepts the value of diversity and thus the significance of learning to work through and across cultures (NASW, 2013), little has been explored in regard to Korean older-adult preferences and expecta- 645 tions concerning the role of physicians in sharing bad news about illness severity. This background would especially assist hospital, hospice, and palliative care social workers in responding with cultural sensitivity to older Korean patients. 650 A major goal of this study was thus to learn about Korean participant preferences concerning disclosure by physicians about terminal illness to the patients and their families.

Favorable attitudes toward the direct dis- 655 closure to patients found in this study were consistent with previous quantitative studies of Korean patients (Yun et al., 2010). The need for full and direct disclosure to patients was noteworthy because of its potential impact on med- 660 ical decisions. Many participants in this study 
believed that direct information would further help patients and family members to make appropriate medical decisions for the given situa-

665 tions. Prolonging life when there is no chance of recovery was thought to be meaningless; hence, accurate information about one's illness would help individuals to make the best end-of-life decision and focus on quality of dying.

670 A subtheme under endorsement of full disclosure using a direct manner included being able to plan one's remaining life in a meaningful way such as completing unfinished business, saying goodbye, and planning for burial. This may be

675 particularly important for those who are in the final stage of life where individuals can review and reflect on their life, strengthen relationships with their loved ones, and plan their death, all of which comprise an integral part of a quality end-

680 of-life experience. Previous studies (Berkman \& Ko, 2010; Jiang et al., 2007) have shown that the patients who were able to complete these tasks positively also looked favorably on the course of their entire life cycle. Concealing informa-

685 tion about the severity of a patient's illness then may be unnecessary, unwanted, and problematic unless this would have a potential benefit that would balance out subterfuge.

Informing patients-providing full informa-

690 tion so that individuals can make their own decisions-was regarded as ethical practice. Unless concealing information can provide a benefit, dishonesty may violate individual autonomy in deciding end-of-life care. Another subtheme

695 relating to the preference for direct disclosure to patients was consideration of patients' coping responses; improved emotional coping by both health care professionals and patients was the expected outcome. Consistent with previous

700 studies (Clayton et al., 2005; Kirk et al., 2004) reporting the importance of physicians' awareness about the negative psychological impact on patients from breaking bad news, these study participants expected that physicians would de-

705 liver bad news in a sensitive manner, including preparing patients and family members for their likely emotional distress prior to breaking such news. Gradual disclosure as a method to minimize potential emotional distress was also iden-

710 tified in previous studies (Clayton et al., 2005; Miyata et al., 2005). Because patients can be easily overwhelmed when too much information is shared at one time in a sensitive topic area such as health status, adjusting the volume of the information and pacing the disclosure will 715 be needed. Not only do health care professionals have responsibility for the way they deliver bad news, but participants also recognized the importance of patients' responsibilities for their own emotional coping and well-being. Accept- 720 ing and confronting the situation, exercising selfcontrol, and developing their own coping strategies may be necessary steps to overcome adverse situations.

The importance of direct disclosure emerged 725 in discussion of end-of-life communication and advanced care planning as a relational process. The majority of the participants wanted their family present when they would be told of their terminal illness. Although family in- 730 volvement in medical decision making is common across cultures, social recognition of the family as an important unit of decision making has long been a tradition in Korean culture. Deeply rooted in Confucianism, family mem- 735 bers, in particular adult children, are expected to be devoted in taking care of their aged parents (Sung, 1995); hence, family involvement in the decision-making process for their loved ones is natural and even expected within the social 740 norm.

The second theme in the present study was about the indirect disclosure of a terminal illness, which features a unique process of information transfer in accord with a high context 745 culture. Although the number of participants in this last group was small, a few preferred disclosing serious illness in an indirect manner. In a high-context culture (Hall, 1976), information is shared through tone of voice, facial expression, 750 gestures, and posture. Communication is generally more indirect and more formal. Through "Noonchi," which means "measuring with the eyes" in Korean (Kim, Kim, \& Kelly, 2006, p. 153), people gain insight about their illness with- 755 out being directly informed. According to a previous study (Yun et al., 2010), 28\% of Korean patients and $23 \%$ of caregivers became aware of the patient's terminal status by the patient's worsening conditions. Unlike the Western cul- 760 ture where values are expressed using explicit 
communication such as language, implicit communication is more easily perceived in some Eastern cultures including the Korean culture (Kwak \& Salmon, 2007). Some participants viewed this practice as beneficial because it minimizes patient emotional distress, but it may create confusion or misunderstanding while individuals try to figure out their illness from implicit cues. It will be important for health care professionals to explore the extent to which patients desire to know about the nature of their illness and accordingly employ tactful communication aligned with their needs.

Another theme in the present study was the preference of nondisclosure to patients. Some participants preferred the information only to be given to the family. This group of participants was concerned that gaining access to information about the terminality of their illness would cause more harm to patients. Notably, they held a fatalistic view that knowing the terminal nature of their illness would not yield any positive effect; rather, it would inflict more pain. This view is contradictory to those who advocate for physicians to tell the truth as the latter approach assists individuals to manage their remaining time effectively. Spending one's remaining lifetime without pain, however, may be the priority over taking care of unfinished business. It is important to recognize that there is variation in how individuals cope with bad news and in their preferences for how to live out their remaining life.

An interesting finding in this study was the perspective that patient and family knowledge about the patient's terminal illness might negatively affect their relationship. Mutual understanding about the patient's illness might provide an opportunity for open communication about end-of-life treatment preferences, but some viewed it as interrupting the natural easygoing interaction between patients and family by creating discomfort. In Korean culture, family members do attempt to conceal bad news to protect the patients from being discouraged - this is not uncommon (Blackhall et al., 2001). Failure to guard loved ones from potential psychological harm might increase guilt and disharmony within the family. This group of participants pre-

810 ferred leaving their decisions to their family to make because their family was deemed able to make the best decisions for them. It should be noted that preferences for family decision making reflect the cultural notion that the family is an extended decision maker, not a disregard for 815 the value of individual autonomy.

\section{SOCIAL WORK IMPLICATIONS}

The results of this study have several implications for social work practice. On an interdisciplinary team, social workers can play a sig- 820 nificant role in providing a holistic approach to treatment. In conducting biospsychosocial assessments, social workers need to assess the patient's understanding about their illness and, where consent is given to speak with family, the 825 family's preferences and concerns about patients being informed of their terminal diagnosis and prognosis. Family might hold different values from the patients due to their concerns about negative psychological effects. It is important 830 that social workers facilitate dialogue between patients and family members to enhance mutual understanding about patient future care (Gutheil \& Heyman, 2006).

It is also important that social workers edu- 835 cate family regarding the importance of understanding patient perspectives and preferences for decision making. Although individual choices for deferring to family decision making should be respected, others who prefer autonomous de- 840 cision making about life-sustaining treatments have the right to voice their concerns and preferences. This is particularly important for those who opt not to rely on life-sustaining treatments, which was a common finding among 845 Koreans (Yun et al., 2011). As found in the current study, the majority of participants preferred that health care professionals provide information about the patients' medical conditions, even if they were incurable. Therefore, helping fam- 850 ily members understand what patients want and what disclosure means to patients and facilitating open communication between patients and family members would maximize mutual understanding about end-of-life care. Preference for 855 disclosure of serious illness varies according to the individual. While attuned to cultural diversity in cancer disclosure especially, social workers 
and other health care professionals must strive to avoid generalizing practice based on stereotyped characteristics of culture (Berkman \& Ko, 2010; Tse, Chong, \& Fok, 2003).

Interdisciplinary approaches to enhance patients' coping can be effective in providing holis-

865 tic care. Assessing individuals' readiness for cancer disclosure, for example, can guide health care professionals, in particular physicians, to tailor their communication approach to individual needs. It is important for health care professionals to assess the extent to which patients are ready to be told, are able to process the information, and would want significant others present in hearing bad news (Crow, Matheson, \& Steed, 2000). Based on an individual's emotional state, the methods for disclosure of a serious illness may need to be tailored. When patients are informed of their illness, psychological supports should be available to patients and family members. Fear of death, being a burden on family, and patients and family members. Providing or linking to the services that provide emotional and spiritual support and creating support groups for family members who are involved in making dif-

885 ficult decisions will be needed. This is especially the case when family members may experience guilt in making decisions for their loved one.

\section{LIMITATIONS, STRENGTHS, AND FUTURE RESEARCH}

This study has several weaknesses. The participants were generally healthy residents in the community instead of people receiving significant health care. Although half of the study participants reported their health status as poor, perspectives toward disclosing terminal illness might be different among those who reside in hospitals or nursing care facilities. As the preference for cancer disclosure might vary by the stage of illness, inclusion of older-adult particitings would also have enriched our understanding.

Another limitation is that the terminology of serious illness we used in this study might
Individuals' preferences for disclosing bad news and to what extent it should be disclosed might be different based on the state and stage of their specific medical condition. Even though patients and family members might want full information 910 about their illness, some may prefer not to know about the prognostic information (Berkman \& Ko, 2010). Taken together, it might be beneficial that future studies assess individual perspectives by diagnosis and prognosis and how they might 915 vary by the stage of the illness. Future research might also look at subpopulations in Korea and other national cultural contexts to learn more about nuances of disclosure of serious illness and decision making. Lastly, we selected the 920 samples from an urban area. There might be a regional difference in attitudes where older adults residing in rural areas might hold different values and beliefs regarding disclosure of terminal illness. Including residents of different regions 925 as participants in future studies would broaden understanding on this topic.

Although there were some limitations listed in this study, there were also some strengths. Prior to this study, there had been no qualitative 930 exploration of Korean older adult views on preferences about delivery of bad news. Although not generalizable, this study offers a nuanced set of viewpoints that highlight the specific role of culture in family communication systems. This 935 type of information is usually inaccessible to social workers yet can provide helpful background in work with Korean older adults and other older Asian populations.

\section{CONCLUSION}

Delivering bad news has important cultural constraints in Korean society as evidenced by the findings in this study. Many of these older Korean study participants favored physicians' disclosure about serious illness to patients, yet 945 thoughts on whether, how, and to whom this information should be disclosed as well as what to consider in delivering bad news varied. Health care professionals thus need to be sensitive to cultural context and individual preferences and 950 needs. 
Because disclosure of serious illness under all circumstances is generally considered the optimal ethical practice in U.S. culture, it is important for health care professionals and others to understand that this practice may not be universally endorsed within certain cultures. However, automatic assumptions about the existence of cultural bias in nondisclosure practice in endof-life situations need to be reexamined in light of cultural norms. Assessing individual patient preferences regarding disclosure of serious illness is an important aspect of end-of-life communication, but one that is even more complex within diverse cultures.

\section{REFERENCES}

Ahrens, T., Yancey, V., \& Kollef, M. (2003). Improving family communications at the end of life: Implications for length of stay in the intensive care unit and resource use. American Journal of Critical Care, 12, 317-324.

Beauchamp, T. L., \& Childress, J. F. (1994). Principles of biomedical ethics. New York, NY: Oxford University.

Berkman, C. S., \& Ko, E. (2009). Preferences for disclosure of information about serious illness among older Korean American immigrants in New York City. Journal of Palliative Medicine, 12, 351-357.

Berkman, C. S., \& Ko, E. (2010). What and when Korean American older adults want to know about serious illness. Journal of Psychosocial Oncology, 28, 244-259.

Blackhall, L., Frank, G., Murphy, S., \& Michel, V. (2001). Bioethics in a different tongue: The case of truth-telling. Journal of Urban Health, 78, 59-71.

Blackhall, L. J., Frank, G., Murphy, S. T., Michel, V., Palmer, J. M., \& Azen, S. P. (1999). Ethnicity and attitudes towards life sustaining technology. Social Science \& Medicine, 48, 1779-1789.

Carrese, J. A., \& Rhodes, L. A. (1995). Western bioethics on the Navajo reservation. Benefit or harm? Journal of the American Medical Association, 274, 826-829.

Clayton, J. M., Butow, P. N., \& Tattersall, M. H. N. (2005). When and how to initiate discussion about prognosis and end-of-life issues with terminally ill patients. Journal of Pain and Symptom Management, 30, 132-144.

Creswell, J., \& Clark, V. L. P. (2007). Designing and conducting mixed methods research. Thousand Oaks, CA: Sage.

Crow, K., Matheson, L., \& Steed, A. (2000). Informed consent and truth-telling: Cultural directions for healthcare providers. The Journal of Nursing Administration, 30, 148-152.
Denzin, N. K., \& Lincoln, Y. S. (2000). Handbook of qualitative research (2nd ed.). Thousand Oaks, CA: Sage.

Elwyn, T. S., Fetters, M. D., Sasaki, H., \& Tsuda, T. (2002). 1005 Responsibility and cancer disclosure in Japan. Social Science \& Medicine, 54, 281-293.

Fielding, R., Wong, L., \& Ko, L. (1998). Strategies of information disclosure to Chinese cancer patients in an Asian community. Psycho-Oncology, 7, 240-251.

Fujimori, M., Akechi, T., Akizuki, N., Okamura, M., Oba, A., Sakano, Y., \& Uchitomi, Y. (2005). Good communication with patients receiving bad news about cancer in Japan. Psycho-Oncology, 14, 1043-1051.

Fujimori, M., Akechi, T., Morita, T., Inagaki, M., Akizuki, 1015 N., Sakano, Y., \& Uchitomi, Y. (2007). Preferences of cancer patients regarding the disclosure of bad news. Psycho-Oncology, 16, 573-581.

Gold, M. (2004). Is honesty always the best policy? Ethical aspects of truth telling. Internal Medicine Journal, 34, 1020 578-580.

Gongal, R. P. R. O. M. (2006). Informing patients about cancer in Nepal: What do people prefer? Palliative Medicine, 20, 471-476.

Gutheil, I. A., \& Heyman, J. C. (2006). They don't want 1025 to hear us: Hispanic elders and adult children speak about end-of-life planning. Journal of Social Work in End-of-Life \& Palliative Care, 2(1), 55-70.

Gwyther, L. P., Altilio, T., Blacker, S., Christ, G., Csikai, E. L., Hooyman, N., ...Howe, J. (2005). Social work 1030 competencies in palliative and end-of-life care. Journal of Social Work in End of-Life \& Palliative Care, 1(1), 87-120.

Hall, E. T. (1976). Beyond culture. New York, NY: Random House.

Jiang, Y., Liu, C., Li, J. Y., Huang, M. J., Yao, W. X., Zhang, R., , \& Wei, Y. Q. (2007). Different attitudes of Chinese patients and their families toward truth telling of different stages of cancer. Psycho-Oncology, 16, 928-936.

Jonsen, A. R., Siegler, M., \& Winslade, W. J. (2010). Clin- 1040 ical ethics: A practical approach to ethical decisions in clinical medicine (7th ed.). New York, NY: McGraw Hill.

Jotkowitz, A., Glick, S., \& Gezundheit, B. (2006). Truthtelling in a culturally diverse world. Cancer Investiga- 1045 tion, 24, 786-789.

Kai, J., Beavan, J., \& Faull, C. (2011). Challenges of mediated communication, disclosure and patient autonomy in cross-cultural cancer care. British Journal of Cancer, 105, 918-924.

Kim, I. J., Kim, L. I. C., \& Kelly, J. G. (2006). Developing cultural competence in working with Korean immigrant families. Journal of Community Psychology, 34, 149-165.

Kirk, P., Kirk, I., \& Kristjanson, L. J. (2004). What do pa- 1055 tients receiving palliative care for cancer and their families want to be told? A Canadian and Australian qualitative study. British Medical Journal, 328, 1134-1441. 
Kwak, J., \& Salmon, J. R. (2007). Attitudes and preferences of Korean-American older adults and caregivers on end-of-life care. Journal of the American Geriatrics Society, 55, 1867-1872.

Lee, J., Keam, B., An, A., Kim, T., Lee, S. H., Kim, D. W., \& Heo, D. S. (2013). Surrogate decision-making in Korean patients with advanced cancer: A longitudinal study. Supportive Care in Cancer, 21, 183-190.

Lincoln, Y., \& Guba, E. (1985). Naturalistic inquiry. New York, NY: Sage

Mauri, E., Vegni, E., Lozza, E., Parker, P., \& Moja, E. (2009). An exploratory study on the Italian patients' preferences regarding how they would like to be told about their cancer. Supportive Care in Cancer, 17, 1523-1530.

Mitchell, J. L. (1998). Cross-cultural issues in the disclosure of cancer. Cancer Practice, 6, 153-160.

Miyata, H., Takahashi, M., Saito, T., Tachimori, H., \& Kai, I. (2005). Disclosure preferences regarding cancer diagnosis and prognosis: To tell or not to tell? Journal of Medical Ethics, 31, 447-451.

1080 Mo, H. N., Shin, D. W., Woo, J. H., Choi, J. Y., Kang, J., Baik, Y. J., , \& Cho, S. H. (2012). Is patient autonomy a critical determinant of quality of life in Korea? End-oflife decision making from the perspective of the patient. Palliative Medicine, 26, 222-231.

1085 National Association of Social Workers. (2013). NASW standards for social work practice in palliative and end of life care. Retrieved from http://www.naswdc.org/ practice/bereavement/standards/default.asp

Oh, D.-Y., Kim, J. E., Lee, C. H., Lim, J. S., Jung, K.H.,

1090 Heo, D. S.,. , \& Kim, N. K. (2004). Discrepancies among patients, family members, and physicians in Korea in terms of values regarding the withholding of treatment from patients with terminal malignancies. Cancer, 100, 1961-1966.

1095 Oliffe, J., Thorne, S., Hislop, T. G., \& Armstrong, E. A. (2007). 'Truth telling' and cultural assumptions in an era of informed consent. Family \& Community Health, $30,5-15$.
Patton, M. Q. (2002). Qualitative research and evaluation methods. Thousand Oaks, CA: Sage.

Shahidi, J. (2010). Not telling the truth: Circumstances leading to concealment of diagnosis and prognosis from cancer patients. European Journal of Cancer Care, 19, 589-593.

Shenton, A. K. (2004). Strategies for ensuring trustwor- 1105 thiness in qualitative research projects. Education for Information, 22, 63-75.

Strauss, A. L., \& Corbin, J. M. (1990). Basics of qualitative research: Ground theory procedures and techniques. Newbury, CA: Sage.

Sung, K. T. (1995). Measures and dimensions of filial piety in Korea. Gerontologist, 35, 240-247.

Surbone, A., Ritossa, C., \& Spagnolo, A. G. (2004). Evolution of truth-telling attitudes and practices in Italy. Critical Reviews in Oncology/Hematology, 52, 165-172. 1115

Tse, C. Y., Chong, A., \& Fok, S. Y. (2003). Breaking bad news: A Chinese perspective. Palliative Medicine, 17, 339-343.

Windsor, J. A., Rossaak, J. I., Chaung, D., Ng, A., Bissett, I. P., \& Johnson, M. H. (2008). Telling the truth to 1120 Asian patients in the hospital setting. The New Zealand Medical Journal, 121(1286), 92-99.

Yun, Y. H., Han, K. H., Park, S., Park, B. W., Cho, C.H., Kim, S., , \& Chun, M. (2011). Attitudes of cancer patients, family caregivers, oncologists and members of 1125 the general public toward critical interventions at the end of life of terminally ill patients. Canadian Medical Association Journal, 183, E673-E679.

Yun, Y. H., Kwon, Y. C., Lee, M. K., Lee, W. J., Jung, K. H., Do, Y. R.,., \& \& Park, S. Y. (2010). Experiences and 1130 attitudes of patients with terminal cancer and their family caregivers toward the disclosure of terminal illness. Journal of Clinical Oncology, 28, 1950-1957.

Yun, Y. H., Lee, C. G., Kim, S. Y., Lee, S. W., Heo, D. S., Kim, J. S., , \& You, C. H. (2004). The attitudes of 1135 cancer patients and their families toward the disclosure of terminal illness. Journal of Clinical Oncology, 22, 307-314. 